\title{
Fabrication of Zero-Speed Sensor Using Weakly Coupled NiFe/CoFe Multilayer Films
}

\author{
Y. Takemura, T. Inoue, M. Nishimoto, and T. Yamada
}

\begin{abstract}
A magnetic sensor consisting of $\mathrm{NiFe} / \mathrm{Al}_{2} \mathrm{O}_{3} / \mathrm{CoFe}$ thin film was fabricated. Magnetization reversal of the NiFe soft layer was asymmetric in switching to parallel and antiparallel alignments to magnetization of $\mathrm{CoFe}$ because of magnetostatic coupling between the NiFe and CoFe layers. When the magnetization of the NiFe was paralleled to that of $\mathrm{CoFe}$, a steep switching accompanied a large Barkhausen jump-induced pulse voltage in a pickup coil as sensor output. Amplitude of the pulse was not dependent on frequency of an external magnetic field. The pulse voltage output was obtained from the sensor element consisting of the optimized film structure excited by the magnetic field at almost zero frequency. These features are also achieved by twisted FeCoV and other wire materials. However, an integrated on-chip sensor with electronic circuits can be fabricated with film-based materials. The film-based sensor elements fabricated in this study can be utilized for speed sensor, rotation sensor, flow meter, and other applications.
\end{abstract}

Index Terms-Large Barkhausen jump, magnetic sensor, thin films.

\section{INTRODUCTION}

A magnetization reversal in magnetic wires with bistable magnetization states generates pulse voltage output in a pickup coil. Amplitude of the voltage does not depend on frequency of an applied magnetic field. The magnetization switching is accompanied with a large Barkhausen jump. Twisted Vicalloy ( $\mathrm{FeCoV}$ alloy) wires have been studied as the optimum material yielding this phenomenon known as the Wiegand effect [1]-[3]. Its switching field of 20-30 Oe is relatively high for sensor applications. Another disadvantage in using the wire is a demagnetizing field arising in the wire, which degrades the sensor performance, especially in short wires. Film-based materials enable a fabrication of a sensor system consisting of a sensor element and an integrated circuit for driving/detecting functions. Although several experimental results have been reported [4]-[6], $\mathrm{NiFe} / \mathrm{CoFe}$ multilayer thin films have been studied in this paper. The combination of an $\mathrm{NiFe}$ soft layer and a $\mathrm{CoFe}$ hard layer has advantages of a large difference in their coercive force and a small switching field of $\mathrm{NiFe}$, which is variable by changing its composition. In order to optimize the magnetostatic coupling between $\mathrm{NiFe}$ and $\mathrm{CoFe}$ layers, an $\mathrm{Al}_{2} \mathrm{O}_{3}$ layer is inserted. Output properties of magnetic field sensors consisting of $\mathrm{NiFe} / \mathrm{Al}_{2} \mathrm{O}_{3} / \mathrm{CoFe}$ multilayer thin films are discussed.

Manuscript received October 16, 2003. This work was supported by The Murata Science Foundation, Japan.

The authors are with Department of Electrical and Computer Engineering, Yokohama National University, Yokohama 240-8501, Japan (e-mail: takemura@ynu.ac.jp).

Digital Object Identifier 10.1109/TMAG.2004.829182

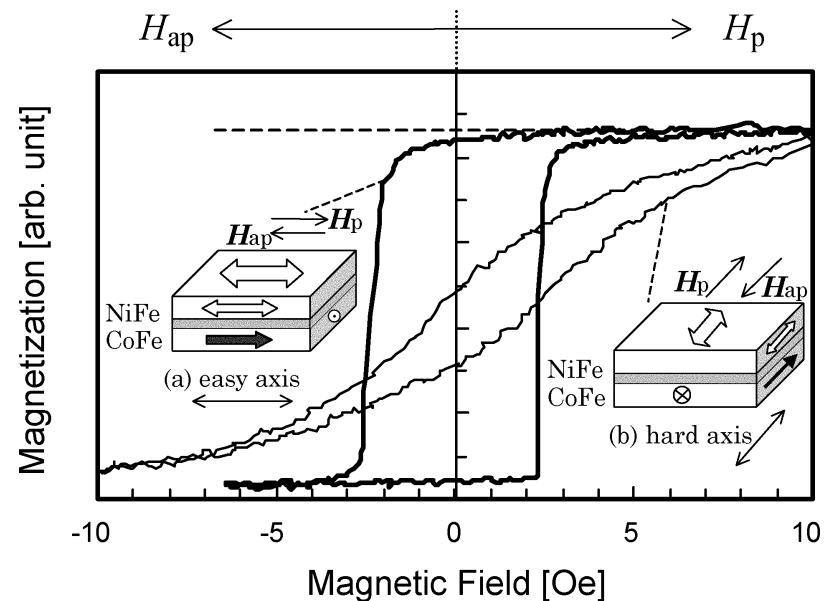

Fig. 1. Minor loops of magnetization curve of $\mathrm{NiFe}(300 \mathrm{~nm}) / \mathrm{Al}_{2} \mathrm{O}_{3}(30 \mathrm{~nm}) / \mathrm{CoFe}(150 \mathrm{~nm})$ thin film. The magnetization of the NiFe soft layer was reversed under the constant magnetization of the $\mathrm{CoFe}$ hard layer. Bold and thin lines indicate minor loops along the (a) easy axis and (b) hard axis of the magnetization anisotropy, respectively.

\section{RESULTS AND DISCUSSION}

\section{A. Sample Preparation and Magnetic Properties}

$\mathrm{NiFe}(300 \mathrm{~nm}) / \mathrm{Al}_{2} \mathrm{O}_{3}(30 \mathrm{~nm}) / \mathrm{CoFe}(150 \mathrm{~nm})$ thin films were prepared on an $\mathrm{SiO}_{2} / \mathrm{Si}$ substrate by rf magnetron sputtering equipment. Uniaxial magnetic anisotropy was induced only in the NiFe layer during the deposition. Magnetization curves of the samples were measured by a vibrating sample magnetometer (VSM) at room temperature. The CoFe layer exhibited an isotropic magnetization along the in-plane direction. A coercive force of the $\mathrm{CoFe}$ layer was 50 Oe. Fig. 1 shows minor loops of the magnetization curves measured by switching a magnetization of the NiFe layer under a constant magnetization of the CoFe layer. A magnetic field of $\boldsymbol{H}_{\mathrm{p}}$ and $\boldsymbol{H}_{\text {ap }}$ indicated in the figure were applied in order to switch the magnetization of the $\mathrm{NiFe}$ parallel and antiparallel to that of the $\mathrm{CoFe}$, respectively. Bold and thin lines indicate the minor loops along the easy and hard axis of magnetic anisotropy of the NiFe layer, respectively. Before each measurement, the magnetization of the CoFe layer was saturated along the applied field direction of $H_{\mathrm{p}}$ for the easy or hard axis of the NiFe layer. The uniaxial magnetic anisotropy of NiFe was clearly observed. The coercive force of NiFe along the easy axis was about 2.5 Oe. Hereafter, only the magnetic properties along the easy axis of $\mathrm{NiFe}$ are discussed. When the magnetization of $\mathrm{NiFe}$ was reversed to the parallel alignment by applying the magnetic field of $\boldsymbol{H}_{\mathrm{p}}$, the magnetization curve exhibited a steep switching, owing to the large Barkhausen effect. The 


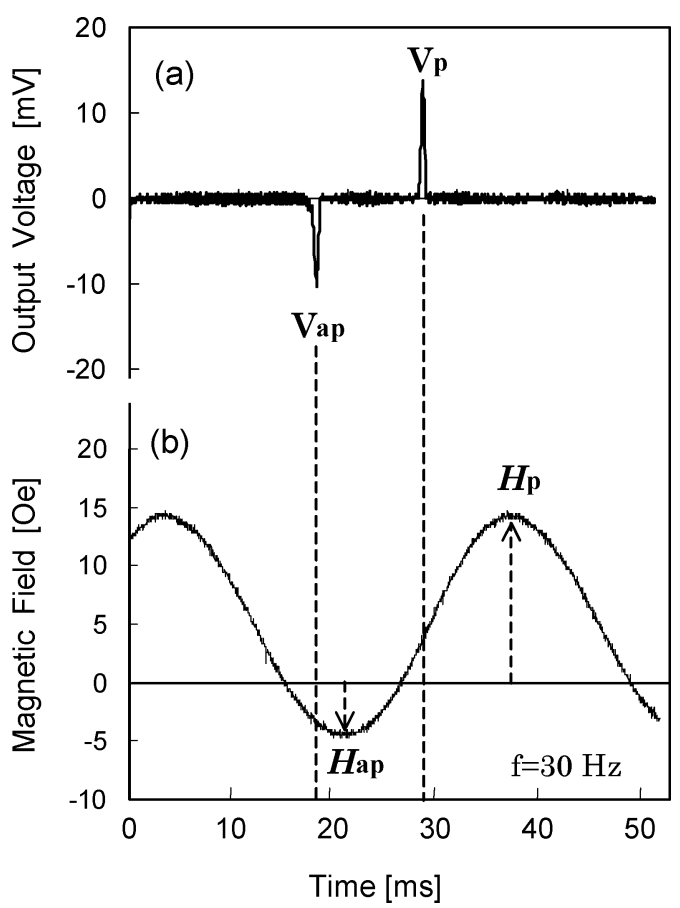

Fig. 2. Waveform of output signal generated from the sensor element consisting of (a) $\mathrm{NiFe} / \mathrm{Al}_{2} \mathrm{O}_{3} / \mathrm{CoFe}$ film and (b) waveform of applied ac magnetic field. $V_{\mathrm{p}}$ and $V_{\text {ap }}$ were observed when the magnetization of $\mathrm{NiFe}$ was reversed to parallel and antiparallel to that of $\mathrm{CoFe}$, respectively. The asymmetric ac magnetic field of $H_{\mathrm{p}}=15 \mathrm{Oe}$ and $H_{\mathrm{ap}}=5 \mathrm{Oe}$ at $30 \mathrm{~Hz}$ was applied.

magnetization reversal of $\mathrm{NiFe}$ from the parallel to antiparallel alignment starts before reaching the remnant state, and it switches gradually. This asymmetric magnetization reversal of $\mathrm{NiFe}$ is attributed to a magnetostatic coupling between the $\mathrm{NiFe}$ and $\mathrm{CoFe}$ layers. These magnetic properties are similar to those of the Wiegand wire [3].

\section{B. Frequency Dependence of Sensor Output}

When the magnetization of $\mathrm{NiFe}$ was reversed parallel to that of $\mathrm{CoFe}$, the pulse voltage owing to the large Barkhausen jump was induced in a pickup coil. The $\mathrm{Al}_{2} \mathrm{O}_{3}$ spacing layer is essential for generating the constant pulse voltage by the applied magnetic field at low frequency down to zero, which is described later. The $\mathrm{Al}_{2} \mathrm{O}_{3}$ thickness of $30 \mathrm{~nm}$ was optimized to adjust the magnetostatic coupling between the $\mathrm{NiFe}$ and $\mathrm{CoFe}$ layers.

Fig. 2 shows waveforms of output signals of the fabricated sensor consisting of the patterned film and a pickup coil of 1000 turns. The 15-mm-long rectangular film (along the easy axis of $\mathrm{NiFe}$ ) by $2 \mathrm{~mm}$ width fabricated by using a metal mask in sputtering deposition was used for the sensor in this measurement. The aspect ratio (=length/width) of this sample was 15:2. When the asymmetric alternating magnetic field of $\boldsymbol{H}_{\mathrm{p}}=15 \mathrm{Oe}$ and $\boldsymbol{H}_{\mathrm{ap}}=5 \mathrm{Oe}$ at $30 \mathrm{~Hz}$ was applied to the sensor, positive and negative output signals indicated as $\boldsymbol{V}_{\mathrm{p}}$ and $\boldsymbol{V}_{\text {ap }}$ in Fig. 2(a) were obtained. $\boldsymbol{V}_{\mathrm{p}}$ and $\boldsymbol{V}_{\text {ap }}$ are associated with parallel and antiparallel magnetization reversals of $\mathrm{NiFe}$, respectively. Fig. 3 shows waveforms of $\boldsymbol{V}_{\mathrm{p}}$ and $\boldsymbol{V}_{\text {ap }}$ measured with various frequency of the applied magnetic field. $V_{\mathrm{p}}$ was stably observed as a single pulse voltage for the entire measured frequency range down to $1 \mathrm{~Hz}$. An area of the pulse (volt-seconds)

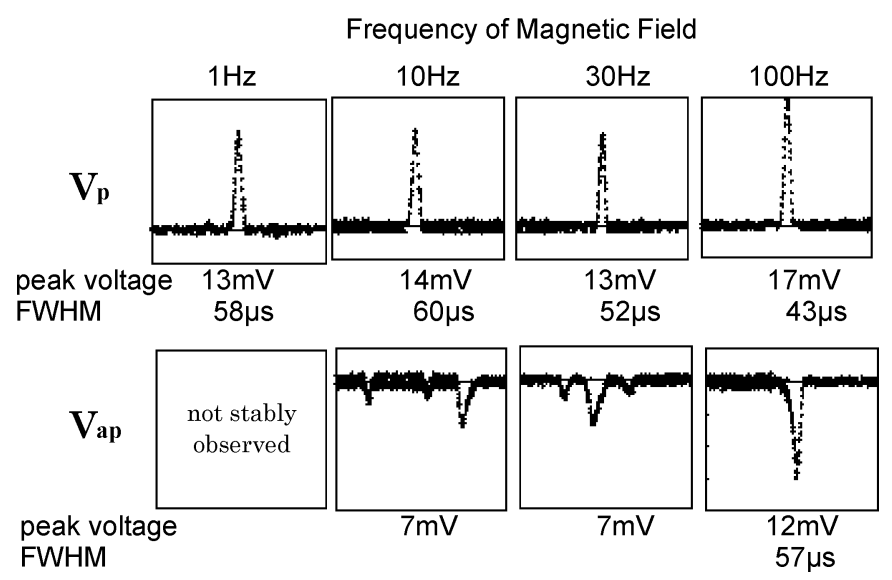

Fig. 3. Waveforms of output voltage signals generated from the sensor element consisting of $\mathrm{NiFe} / \mathrm{Al}_{2} \mathrm{O}_{3} / \mathrm{CoFe}$ film. The asymmetric ac magnetic field of $H_{\mathrm{p}}=15 \mathrm{Oe}$ and $H_{\mathrm{ap}}=5 \mathrm{Oe}$ at a frequency of $1-100 \mathrm{~Hz}$ was applied.

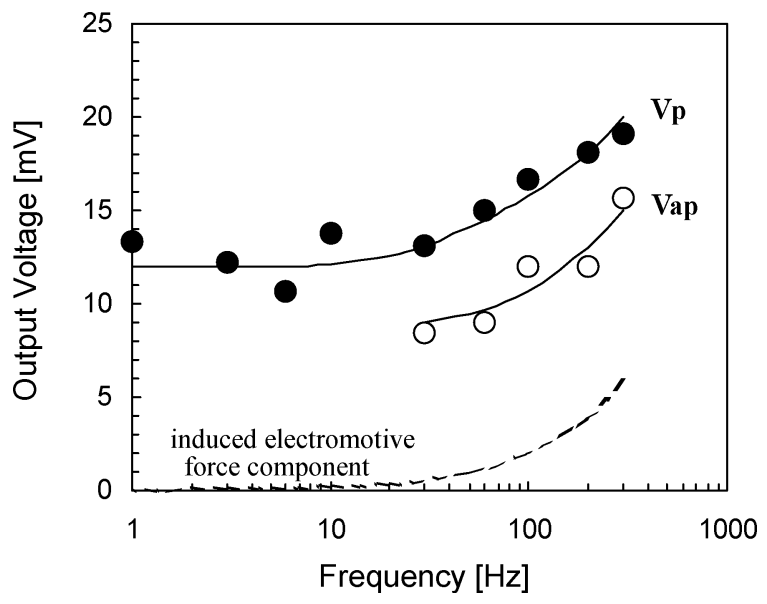

Fig. 4. Frequency dependence of amplitude of output pulse voltage generated from the sensor element consisting of $\mathrm{NiFe} / \mathrm{Al}_{2} \mathrm{O}_{3} / \mathrm{CoFe}$ film. The asymmetric ac magnetic field of $H_{\mathrm{p}}=15 \mathrm{Oe}$ and $H_{\mathrm{ap}}=5$ Oe was applied.

agreed with the calculated value, assuming the saturation flux density $\boldsymbol{B}_{\mathrm{s}}=1.05 \mathrm{~T}$ of NiFe. The waveform of $\boldsymbol{V}_{\text {ap }}$ exhibited a single peak at a high frequency of $100 \mathrm{~Hz}$ but split in several peaks with their time differential of within $0.3 \mathrm{~ms}$ at lower frequency. It was because that the magnetization reversal of $\mathrm{NiFe}$ in antiparallel process was discontinuous and was not attributed to the large Barkhausen effect. Full width at half maximum (FWHM) of $\boldsymbol{V}_{\mathrm{p}}$ was narrower than that of $\boldsymbol{V}_{\mathrm{ap}}$.

The amplitude of the output signals was plotted as a function of frequency of the applied magnetic field in Fig. 4. The amplitude of $V_{\mathrm{p}}$ was constant at around $12 \mathrm{mV}(1.2 \mu \mathrm{V}$ per turn of the pickup coil) at the frequency range from 1 to $30 \mathrm{~Hz}$, which suggested that the magnetization reversal of NiFe to the parallel alignment was attributed to the large Barkhausen effect. $V_{\text {ap }}$ was not stably measured at frequency lower than $20 \mathrm{~Hz}$. $V_{\mathrm{p}}$ was larger than $V_{\text {ap }}$ at any frequency. Both $V_{\mathrm{p}}$ and $V_{\text {ap }}$ increased with increasing frequency because of the electromotive force induced in the coil by the ac magnetic field.

\section{Aspect Ratio of Magnetic Films}

In order to reduce the size of the sensor, films of $6 \mathrm{~mm}$ in length were used. The films were originally patterned to a round 


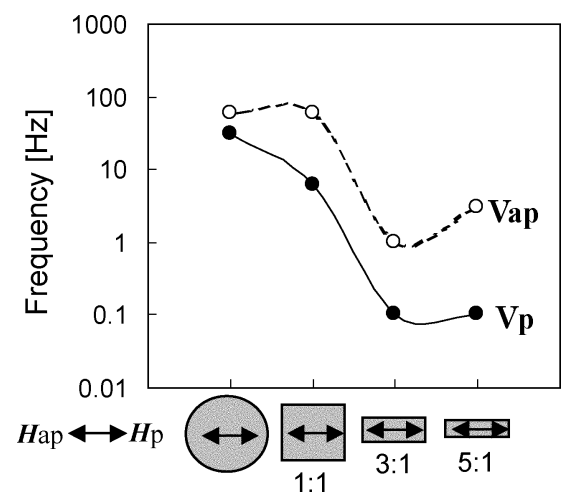

Fig. 5. Lowest frequency at which output voltage was observed as a single pulse from the sensor elements consisting of $\mathrm{NiFe} / \mathrm{Al}_{2} \mathrm{O}_{3} / \mathrm{CoFe}$ films. The symmetric ac magnetic field of $H_{\mathrm{p}}=H_{\mathrm{ap}}=5$ Oe was applied. The films were patterned from circles and rectangles with various aspect ratios.

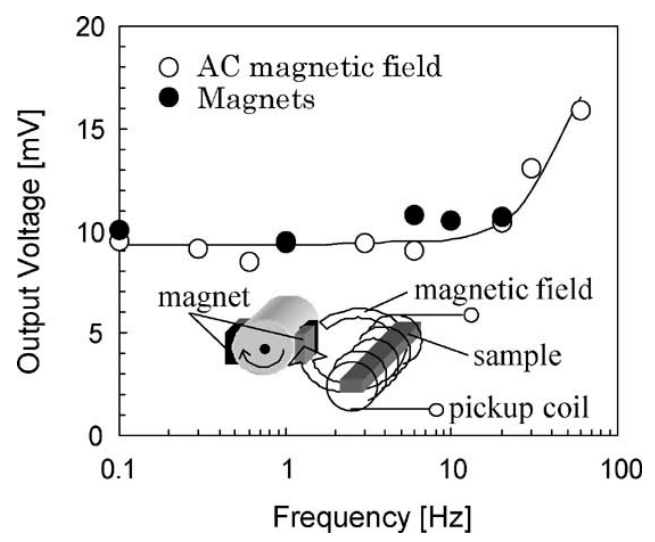

Fig. 6. Frequency dependence of output pulse voltage generated from the sensor element consisting of $\mathrm{NiFe} / \mathrm{Al}_{2} \mathrm{O}_{3} / \mathrm{CoFe}$ film. The symmetric ac magnetic field of $H_{\mathrm{p}}=H_{\mathrm{ap}}=5$ Oe or the magnetic field from a pair of permanent magnets was applied.

shape by using a metal mask during the deposition. After the deposition, they were patterned to rectangles with various aspect ratio of length/width by photolithography and wet etching processes. The length along the easy axis of magnetization was constant at $6 \mathrm{~mm}$ for all the samples and the width was varied. A frequency dependence of output voltage of sensors using these films was measured. Fig. 5 shows the lowest frequency at which the output signal was observed as a single pulse voltage. In this measurement, a symmetric sine-wave ac magnetic field of $\boldsymbol{H}_{\mathrm{p}}=\boldsymbol{H}_{\mathrm{ap}}=5$ Oe was applied to the sensors. An asymmetric ac magnetic field has been traditionally used in measurements of Wiegand wires in order to generate higher output voltage [3], [4], [7], but the symmetric ac magnetic field is preferred for actual sensor applications. For the as-deposited round film, both $\boldsymbol{V}_{\mathrm{p}}$ and $\boldsymbol{V}_{\text {ap }}$ were observed at higher frequency than $30 \mathrm{~Hz}$.
The pulse voltage of $V_{\mathrm{p}}$ was generated at lower frequency from the square sample (rectangle of aspect ratio 1:1) compared with the round sample, which was explained by an existence of edge magnetic domain structures in the square film. For the rectangular samples of aspect ratio of 3:1 and 5:1, $\boldsymbol{V}_{\mathrm{p}}$ was observed as a single pulse voltage at low frequency down to $0.1 \mathrm{~Hz}$. By increasing the aspect ratio of length/width of samples, the frequency range for observing a single pulse voltage was lowered. The samples with the aspect ratio higher than $3: 1$, in which the demagnetizing field was relatively small, exhibited a steep magnetization switching with a large Barkhausen jump.

\section{Sensor Output Driven by Permanent Magnets}

Fig. 6 shows the frequency dependence of the amplitude of $\boldsymbol{V}_{\mathrm{p}}$ obtained from the sensor consisting of the film whose aspect ratio was 5:1. The symmetric ac magnetic field of $\boldsymbol{H}_{\mathrm{p}}=\boldsymbol{H}_{\mathrm{ap}}=$ 5 Oe was applied. The amplitude of the voltage was constant at $10 \mathrm{mV}(10 \mu \mathrm{V} /$ turn $)$ for the frequency range from 0.1 to $30 \mathrm{~Hz}$. The output voltage was also measured from the sensor, driven by a pair of permanent magnets equipped on a rotor, as indicated in the insertion of Fig. 6. The amplitude of $\boldsymbol{V}_{\mathrm{p}}$ excited by the magnets (solid circles in the figure) was the same as that of the sine-wave ac magnetic field.

\section{CONCLUSION}

We have studied $\mathrm{NiFe} / \mathrm{Al}_{2} \mathrm{O}_{3} / \mathrm{CoFe}$ multilayer films with small switching field as a candidate for film-based zero-speed sensor. The fabricated film-based sensor elements generate output voltages of a single pulse by detecting a magnetic field higher than 5 Oe. The amplitude and width of the pulse is not dependent on frequency of the field down to $0.1 \mathrm{~Hz}$, which may not be achieved by a Hall sensor, magnetoresistive (MR) sensor, or a conventional inductive sensor.

\section{REFERENCES}

[1] J. R. Wiegand and M. Velinsky, U.S. Patent 3820 090, 1974.

[2] P. E. Wigen, "Wiegand wire: New material for magnetic-based devices," Electron., vol. 48, pp. 100-105, 1975.

[3] S. Abe, A. Matsushita, and M. Naoe, "Dependence of large Barkhausen jump on length of a Vicalloy fine wire with torsion stress," IEEE Trans. Magn., vol. 34, pp. 1318-1320, July 1998.

[4] S. Abe, A. Matsushita, K. Negishi, Y. Baba, and M. Naoe, "Generation of large Barkhausen jump in bilayered thin film," IEEE Trans. Magn., vol. 35, pp. 3634-3636, Sept. 1999.

[5] N. Yano, S. Furukawa, K. Oka, I. Ogasawara, J. Yamasaki, and F. B. Humphrey, "Large Barkhausen discontinuity of Co-Fe-Si-B amorphous films sputtered on polymer substrate," IEEE Trans. Magn., vol. 32, pp. 4532-4534, Sept. 1996.

[6] V. I. Malyutin, "New high-speed planar-type thin film pulse magnetic elements," in INTERMAG Dig.BQ-14, Seattle, WA, 1996.

[7] Y. Takemura and A. Matsushita, "Frequency dependence of output voltage generated from bundled compound magnetic wires," IEEE Trans. Magn., vol. 37, pp. 2862-2864, July 2001. 